Pacific Journal of Mathematic 


\title{
ORTHOGONAL GROUPS OF DYADIC UNIMODULAR QUADRATIC FORMS II
}

\author{
D. G. JAMES
}

\begin{abstract}
Let $O(M)$ be the orthogonal group of a unimodular quadratic form over the integers in a dyadic local field. The subgroups of $O(M)$ normalized by the commutator subgroup are classified when the rank $r(M) \geqq 9$, or when $r(M) \geqq 7$ and the residue class field has at least 8 elements.
\end{abstract}

Classifications of the subgroups of an orthogonal group normalized by the commutator subgroup have been given by many authors. For isotropic nonsingular quadratic forms over fields there is the fundamental result of Dickson [3] and Dieudonné [4]: The projective commutator subgroup is simple when the form has dimension at least 5. Other proofs of this, which allow the field to have characteristic two, have been given by Eichler [5] and Tamagawa [17]. In [12], Klingenberg generalized this result to nondegenerate quadratic forms over local rings, provided the residue class field is not of characteristic two, and classified the subgroups normalized by the commutator subgroup by using congruence subgroups and mixed commutator subgroups. Klingenberg's work has been further extended in [1, 2, $7-10,13,16,18,19]$ by relaxing the restrictions either on the form or on the ring. In particular, I studied this problem for unimodular quadratic forms over the ring of integers in a dyadic local field with 2 an unramified prime and the residue class field having at least 8 elements $[9,10]$. These last two restrictions will now be removed, that is, 2 may ramify and there is no restriction on the residue class field (except only that it is perfect).

An outline of the paper follows. Denote by o the ring of integers in a dyadic local field $F$ and by $M$ a free o-module of finite rank $r(M) \geqq 3$ endowed with an isotropic symmetric bilinear form $B: M \times$ $M \rightarrow \mathfrak{D}$ with determinant a unit in $\mathfrak{D}$. After introducing some basic isometries, the commutator subgroup $\Omega(M)$ of the orthogonal group $O(M)$ is determined. Apart for a few exceptional modules $M$ with small rank, $\Omega(M)$ is equal to the spinorial kernel of $O(M)$ and is generated by the Siegel transformations. Next, the "primitive" submodules $M_{\xi}, \xi \in \Xi(\Xi$ a suitable indexing set), invariant under the action of the commutator subgroup are determined. For each ideal $\mathfrak{a}$ in $\mathfrak{D}$, the submodules $\mathfrak{a} M_{\xi}$ are still invariant and are used to define the subgroups $\mathscr{E}\left(a M_{\xi}\right)$ and $\mathscr{F}\left(\mathfrak{a} M_{\xi}\right)$. The main result is:

$$
\text { If } r(M) \geqq 9, \text { a subgroup } \mathscr{N} \text { of the orthogonal group } O(M) \text { is }
$$


normalized by the commutator subgroup $\Omega(M)$ if and only if it satisfies a ladder relation of the form

$$
\mathscr{E}\left(\mathfrak{a} M_{\xi}\right) \subseteq \mathscr{N} \subseteq \mathscr{F}\left(\mathfrak{a} M_{\xi}\right)
$$

for some ideal a in $\mathfrak{o}$ and some $\xi \in \Xi$.

The restriction $r(M) \geqq 9$ can be weakened to $r(M) \geqq 7$ if the residue class field has at least 8 elements. If $a q\left(M_{\xi}\right) \subseteq 0$, the subgroups $\mathscr{E}\left(a M_{\xi}\right)$ can be characterized as mixed commutator subgroups with the help of congruence subgroups obtained from $a M_{\xi}$. In a subsequent paper we shall indicate how the local structure obtained here can be injected into orthogonal groups over Dedekind domains. In particular, some of the structure of $\mathscr{F}\left(a M_{\xi}\right) / \mathscr{E}\left(a M_{\xi}\right)$ that transfers to the global situation will be given.

The notation for subgroups in $[9,10]$ has been slightly modified in the present paper. In particular, $\mathscr{E}_{*}(a)$ will now be written as $\mathscr{E}\left(\mathfrak{a} M_{*}\right)$ and the subgroups $\mathscr{E}(\mathfrak{a}, \zeta)$ are now included amongst those denoted $\mathscr{E}\left(a M_{\xi}\right)$. Similarly, $\mathscr{F}^{*}(\mathfrak{a})$ becomes $\mathscr{F}\left(\mathfrak{a} M^{*}\right)$ and corresponding changes will be made for the congruence subgroups.

1. Preliminaries. Let $V$ be a finite dimensional vector space over the dyadic local field $F$ of characteristic zero and $q: V \rightarrow F$ a quadratic form on $V$, that is, $q(\alpha x)=\alpha^{2} q(x)$ for $\alpha \in F, x \in V$ and the symmetric mapping $B: V \times V \rightarrow F$ defined by

$$
B(x, y)=q(x+y)-q(x)-q(y)
$$

is bilinear. Denote by 0 the ring of integers in $F$, by $\mathfrak{p}$ the maximal ideal in $\mathfrak{D}$ and by $\mathfrak{H}$ the group of units. Assume $V$ supports a unimodular lattice $M$; thus $M$ is a free o-module spanning $V$ over $F$ with $B(M$, $M)=\mathfrak{p}$ and $\operatorname{det}_{B}(M)$ a unit. Unimodular lattices are discussed in [14; §93D]; we summarize below the main results required.

Fix a prime $\pi$ in 0 and a normalized valuation ord on $F$. Thus ord $\pi=1$ and ord $2=e \geqq 1$. In

$$
q(M)=\{q(x) \mid x \in M\} \subseteq \frac{1}{2} \mathfrak{D}
$$

choose $q(w)=(1 / 2) a$ such that ord $a$ is minimal. O'Meara calls $a$ a norm generator of $M$. The norm group is

$$
\mathfrak{g} M=2(q(M)+\mathfrak{p}) .
$$

Let $\mathfrak{m} M$ denote the largest ideal of $\mathrm{o}$ in $\mathrm{g} M$ and define the weight $\mathfrak{w} M$ by the equation

$$
\mathfrak{w} M=\mathfrak{p} \mathfrak{m} M+2 \mathfrak{o} \text {. }
$$


Then $b \in \mathfrak{o}$ is called a weight generator of $M$ if $b \mathfrak{o}=\mathfrak{w} M$.

If $r(M) \geqq 5$, or if $r(M) \geqq 3$ and ord $(a b)$ is even, $M$ is split by a hyperbolic plane. Thus

$$
M=H \perp K
$$

where $H=\mathrm{o} u+\mathrm{o} v$ with $q(u)=q(v)=0$ and $B(u, v)=1$. In this manner we can reduce the general unimodular lattice to the form

$$
M=H \perp N \perp L
$$

where $r(L) \leqq 4$ and $q(N) \leqq \mathfrak{D}$. Here $N$ will be an orthogonal sum of hyperbolic planes plus, possibly, the anisotropic binary plane $\langle A(2$, $2 \rho)\rangle$. In general, $\langle A(\alpha, \beta)\rangle$ denotes a binary unimodular lattice $\mathrm{o} x+$ o $y$ where $2 q(x)=B(x, x)=\alpha, 2 q(y)=B(y, y)=\beta$ and $B(x, y)=1$. For $\alpha \in \mathfrak{t}$, denote by $\langle\alpha\rangle$ a lattice $\mathfrak{o} x$ where $B(x, x)=\alpha$. If $r(L) \geqq 1$, the lattice $L$ obtained above in the splitting of $M$ can be taken as one of the forms given in the following table. Here $a$ and $b$ are norm and weight generators, ord $c \geqq$ ord $b$ and $\zeta \in \mathfrak{D}$ (see $[14 ; 93: 17-18]$ ). Moreover, when $r(L)=2$ and ord $(a b)$ is even, we may take $b=2$. It will be apparent later that the subgroup structure of the orthogonal group $O(M)$ is determined mainly by $L$.

\begin{tabular}{|c|c|}
\hline$r(L)$ & $L$ \\
\hline 1 & $\mathrm{D} w=\langle a\rangle$ \\
\hline 2 & $\mathrm{o} w+\mathrm{o} z=\langle A(a, c)\rangle$ \\
\hline 3 & $\mathfrak{v} w \perp(\mathrm{o} x+\mathrm{o} y)=\langle a\rangle \perp\langle A(b, 2 \zeta)\rangle$ \\
\hline 4 & $(\mathfrak{o} w+\mathrm{o} z) \perp(\mathrm{o} x+\mathfrak{o} y)=\langle A(a, c)\rangle \perp\langle A(b, 2 \zeta)\rangle$ \\
\hline
\end{tabular}

TABLE I

Denote by $M_{*}$ the sublattice of $M$ consisting of all $r \in M$ with $q(r)$ in $\mathfrak{D}$. Let $p$ and $r$ in $M_{*}$ be such that $q(p)=B(p, r)=0$. Then $E(p, r)$ denotes the Siegel transformation defined for $s \in M$ by

$E(p, r)(s)=s-B(p, s) r+B(r, s) p-q(r) B(p, s) p$.

Now assume that $M$ is split by a hyperbolic plane $H=\mathfrak{D} u+\mathfrak{D} v=$ $\langle A(0,0)\rangle$. In future $H$ denotes this fixed hyperbolic plane. Then $M=H \perp K$ with $K$ unimodular. Denote by $\mathscr{E}$ the subgroup of $O(M)$ generated by the Siegel transformations $E(u, r)$ and $E(v, r)$ with $r$ ranging over $K_{*}=K \cap M_{*}$. The isometries $\Delta$ and $\Phi(\varepsilon)$, where $\varepsilon$ is a unit, are defined by

$$
\Delta: u \longmapsto v, v \longmapsto u, s \longmapsto s \text { for } s \in K
$$

and

$$
\Phi(\varepsilon): u \longmapsto \varepsilon u, v \longmapsto \varepsilon^{-1} v, s \longmapsto s \text { for } s \in K
$$


Finally, if $r \in M$ is such that $q(r) \notin \mathfrak{p}$, denote by $\Psi(r)$ the symmetry about $r$ defined by

$$
\Psi(r)(s)=s-q(r)^{-1} B(r, s) r .
$$

Proposition 1.1. The following relations hold.

$$
\Delta \Phi(\varepsilon) \Delta^{-1}=\Phi\left(\varepsilon^{-1}\right) .
$$

For $\theta \in O(M), q(p)=B(p, r)=0$ and $r \in M_{*}$

$$
\theta E(p, r) \theta^{-1}=E(\theta(p), \theta(r)) \text {. }
$$

In particular,

$$
\begin{aligned}
\Delta E(u, r) \Delta^{-1} & =E(v, r) \\
\Phi(\varepsilon) E(u, r) \Phi\left(\varepsilon^{-1}\right) & =E(u, \varepsilon r) \\
\Phi(\varepsilon) E(v, r) \Phi\left(\varepsilon^{-1}\right) & =E\left(v, \varepsilon^{-1} r\right) .
\end{aligned}
$$

Also, for $r, s \in M_{*}$ with $B(p, r)=B(p, s)=0$,

$$
E(p, r) E(p, s)=E(p, r+s) \text {. }
$$

Proof. These are well-known and easy to verify.

Perhaps less well-known are the following two identities.

Proposition 1.2. Let $M=H \perp K$. Let $r \in K_{*}$ and $\beta \in \mathfrak{o}$ be such that $\varepsilon=1-\beta q(r)$ is a unit. Then

$$
E(v, \beta r) E(u, r)=E\left(u, \varepsilon^{-1} r\right) E(v, \beta \varepsilon r) \Phi\left(\varepsilon^{-2}\right) .
$$

Let $s \in K$ have $q(s)$ a unit. Then

$$
\Delta \Psi(s)=\Phi(-q(s)) E(v, s) E\left(u, q(s)^{-1} s\right) E(v, s) .
$$

Proof. These can be verified by checking the images of $u, v$ and $t \in K$. Alternatively (see [10]), they can be established by making suitable calculations in the Clifford algebra of $V$.

Corollary 1.3. Let $M=H \perp K$ and $q(K)$ contain a unit. Then for all units $\varepsilon$ in $\mathfrak{u}, \Phi\left(\varepsilon^{2}\right) \in \mathscr{E}$. In particular, the hypothesis is satisfied if $r(M) \geqq 5$.

Proof. Take $r \in K$ with $q(r)$ a unit and put $\beta=q(r)^{-1}(\varepsilon-1)$. The result now follows from the first identity in Proposition 1.2. If $r(K) \geqq 3$ and ord $(a b)$ is even, then $K$ is split by a hyperbolic plane and consequently represents units. If ord $(a b)$ is odd, either 
ord $\left(2 a^{-1}\right)$ or ord $\left(2 b^{-1}\right)$ is even, and $K$ clearly represents units.

The characteristic set $\mathscr{L}(s)$ of a primitive element $s$ in $M$ is defined by

$$
\mathscr{C}(s)=\{t \in M \mid B(s, t)=1\} .
$$

Since $M$ is unimodular, $\mathscr{\mathscr { C }}(s)$ is not empty. For any isometry $\varphi \in$ $O(M)$,

$$
q(\mathscr{L}(\varphi(s))) \equiv q(\mathscr{C}(s)) \bmod \mathrm{D} .
$$

This relation controls the equivalence of elements of $M$ under the action of the orthogonal group (see Hsia [6]).

If $M=M_{1} \perp M_{2}$, denote by $O\left(M_{1}\right)$ the subgroup of isometries in $O(M)$ that act identically on $M_{2}$. Let $i(M)$ denote the Witt index of $M$.

Proposition 1.4. Let $M=H \perp K$ and $q(K)$ contain a unit. If card $\mathrm{o} / \mathfrak{p}=2$, assume also $r(M) \geqq 7$, or $i(M)=1$, or $M=H \perp H^{\prime} \perp K^{\prime}$ and $q\left(K^{\prime}\right)$ contains a unit. Then for each $\varphi \in O(M)$ there exists an isometry $\psi \in \mathscr{E}$ such that

$$
\psi \varphi \psi^{-1}=E(u, x) E(v, y) \Phi(\varepsilon) \theta
$$

where $x, y \in K_{*}, \varepsilon$ is a unit and $\theta \in O(K)$.

Proof. The proof of Lemma 3.6 (3), (4) in [9] is modified as follows.

(3) Assume $\alpha, \beta \in \mathfrak{p}$. Then $s$ is primitive in $K$. The characteristic set of $v$ is

$$
\mathscr{M}(v)=\{z \in M \mid B(v, z)=1\}=u+(K \perp \mathrm{o} v) .
$$

Since $\varphi(\mathscr{M}(v))=\mathscr{M}(\varphi(v))$, there exists $t_{1} \in \mathscr{M}(\varphi(v))$ such that $q\left(t_{1}\right)$ is a unit. Let $t$ be the component of $t_{1}$ in $K$. Then $q(t) \in \mathfrak{D}$. Also, since $B\left(t_{1}, \varphi(v)\right)=1$ and $\alpha, \beta \in \mathfrak{p}$, it follows that $B(s, t)$ is a unit. Hence $B(s, t)+\alpha q(t)$ is a unit.

(4) Finally assume $\alpha$ is a unit and $\beta \in \mathfrak{p}$. If card $\mathfrak{o} / \mathfrak{p} \geqq 4$, the earlier version still holds. There remains the case card $\mathfrak{v} / \mathfrak{p}=2$ and $B(s, t)$ a unit. Then $K=(\mathfrak{o s}+\mathfrak{o} t) \perp K^{\prime}$. If $q(s)$ is a unit, replace $t$ by $s$. Otherwise os + ot is a hyperbolic plane $H^{\prime}$. Now choose a new $t \in K^{\prime}$ with $q(t)$ a unit and $B(s, t)=0$. This completes the proof.

2. Generators for $O(M)$. In this section we obtain generators for the orthogonal group $O(M)$ (see also O'Meara and Pollak [15]). 
Proposition 2.1. Let $M=H \perp N \perp L$ where $q(N) \subseteq$ D. Then the orthogonal group $O(M)$ is generated by $\mathscr{E}$ and $O(H \perp L)$.

Proof. The proof of Theorem 2.1(1) in [9] or of the lemma in [10; IV] generalizes without significant change.

REMARK 2.2. Let $w, z \in L$ be such that $B(w, z)=1$ and $q(z) \in \mathfrak{D}$ (but not necessarily $q(w) \in \mathfrak{p}$ ). The argument in [9; Theorem 2.1(1)] also shows that $\varphi \in O(H \perp L)$ can be changed by isometries in $\mathscr{E}$, and $\Delta$, to an isometry fixing $w$. This fact will be used later.

It is clear that $O(H)$ is generated by $\Delta$ and the isometries $\Phi(\varepsilon)$. We now obtain generators for $O(H \perp L)$ where $L$ is as in Table I.

2.3. Let $L=\mathfrak{D} w=\langle a\rangle$. Then $O(M)$ is generated by $O(H)$ and $\mathscr{E}$, together with $\Psi(w)$ if 2 is tamely ramified (ord 2 odd).

Proof. By Proposition 2.1 it suffices to consider $O(H \perp \mathrm{o} w)$. Let $\varphi \in O(H \perp \mathfrak{o} w)$ and

$$
\varphi(w)=\beta u+\gamma v+\delta w
$$

where $\delta$ is a unit. Then

$$
(\mathfrak{D} \varphi(w))^{\perp}=\mathfrak{D}(\alpha \delta v-\beta w)+\mathfrak{o}(\alpha \delta u-\gamma w) \cong H .
$$

It follows that $q(\beta w)$ and $q(\gamma w)$ are in $\mathrm{o}$. Assume $2 \in \mathfrak{D}(1-\delta)$ (a similar argument will hold if instead $2 \in \mathfrak{p}(1+\delta))$. Using

$$
\beta \gamma=\frac{1}{2} a\left(1-\delta^{2}\right)
$$

it follows that

$$
E\left(u, \gamma^{-1}(1+\delta) w\right) \varphi(w)=\gamma v-w
$$

Then

$$
\Psi(w) E\left(v, a^{-1} \gamma w\right)(\gamma v-w)=w,
$$

and we have reduced $\varphi$ to an isometry in $O(H)$. When 2 is wildly ramified, $\Psi(w)$ can be expressed in terms of the isometries in $O(H)$ and $\mathscr{E}$ using the second identity in Proposition 1.2.

2.4. Let $L=\mathfrak{o} w+\mathfrak{o} z=\langle A(a, c)\rangle$. Then $O(M)$ is generated by $O(H)$ and $\mathscr{E}$, together with $\Psi(w-a z)$ if ord $(2 a)$ is odd, and with $\Psi(z)$ if ord $\left(2 c^{-1}\right)$ is odd and positive.

Proof. We first change $\phi \in O(M)$ by the given isometries to an 
isometry fixing $w$. If $q(z)=(1 / 2) c \in \mathfrak{D}$, Remark 2.2 gives this immediately. We therefore assume ord $(a c)$ is odd (otherwise, change $z$ and increase ord $c$ ). Again, by Proposition 1.4, since $q(L)$ now contains a unit, assume $\varphi \in O(L)$.

Let ord $\left(2 c^{-1}\right)=g \geqq 1$ and $h=[(g+1) / 2]$ (integral part). Then $q\left(\pi^{h} z\right) \in \mathcal{D}$ and $E\left(u, \pi^{h} z\right)(w)=\pi^{h} u+w$. Let

$$
\varphi\left(\pi^{h} u+w\right)=\pi^{h} u+w+\lambda w+\mu z .
$$

If $x=\pi^{-h}(\lambda w+\mu z)$ is in $L_{*}$, replacing $\varphi$ by

$$
\psi=E\left(u,-\pi^{h} z\right) E(v, x) \varphi E\left(u, \pi^{h} z\right)
$$

gives the desired reduction since $\psi(w)=w$.

From $q(w)=q\left(\varphi\left(\pi^{h} u+w\right)\right)$, it follows that

$$
\frac{1}{2} a \lambda^{2}+\frac{1}{2} c \mu^{2}=-a \lambda-(\lambda+1) \mu \in \mathfrak{D}
$$

and hence, since ord $(a c)$ is odd, $l=$ ord $\lambda \geqq(1 / 2)$ ord $\left(2 a^{-1}\right)$ and $m=$ ord $\mu \geqq h \geqq(1 / 2) g$. Let $f$ be the minimum order of the four terms in this equation, that is

$$
f=\min \left\{2 l-\operatorname{ord}\left(2 a^{-1}\right), 2 m-g, l+\operatorname{ord} a, m\right\} .
$$

Assume $f<g$. If $f=m$, then $2 m-g \geqq m$ leads to a contradiction. Similarly, $f=l+$ ord $a$ gives a contradiction with $2 l-$ ord $\left(2 a^{-1}\right) \geqq$ $f$. Since there must be at least two terms with the minimum order, this leaves

$$
f=2 m-g=2 l-\operatorname{ord}\left(2 a^{-1}\right)
$$

which contradicts the hypothesis that ord (ac) is odd. Hence $f \geqq g$. This will now be strengthened to $f \geqq 2 h$, which ensures that $x \in L_{*}$ as required.

If $g$ is even, $2 h=g$. Assume, therefore, $g=2 h-1$ and $f=g$. Considering again the definition of $f$, both $f=l+$ ord $a$ and $f=$ $2 l$ - ord $\left(2 a^{-1}\right)$ (which is even) lead to contradictions. Hence $f=$ $m=2 m-g=g$ and $(1 / 2) c \mu+\lambda+1 \equiv 0 \bmod \pi$. Replace $\varphi$ by $\Psi(z) \varphi$ and the new coefficient of $z$ lies in $\pi^{g+1} \mathfrak{D}$. Repeating the previous calculations now gives $f \geqq g+1=2 h$.

We may now assume $\varphi(w)=w$. Modifying the argument in 2.3, we now reduce $\varphi$ to an isometry in $O(H)$. Let $r=w-a z$ so that $B(r, w)=0$. Since $B(\varphi(z), w)=1$,

$$
\varphi(z)=\alpha u+\beta v+\gamma r+z
$$

for some $\alpha, \beta, \gamma \in \mathfrak{D}$. Computing characteristic sets gives 


$$
\mathscr{M}(z)=w+H \perp \mathfrak{o}(z-c w)
$$

and

$$
2 q(\mathscr{M}(\varphi(z))) \equiv 2 q(\mathscr{M}(z)) \equiv\left\{a+\rho^{2} c(1+a c) \mid \rho \in \mathfrak{p}\right\} \bmod 2 \mathfrak{o} .
$$

Since $v+(1-\alpha) w \in \mathscr{M}(\varphi(z))$, and either $c \in 20$ or ord $(a c)$ is odd, it follows that $a \alpha^{2} \in 20$. Similarly, $a \beta^{2} \in 20$. Let $\sigma=-B(r, z)-\gamma q(r)$. Then $q(z)=q(\varphi(z))$ gives $a \gamma \sigma=a \alpha \beta \in 20$. But ord $(\gamma q(r))=\operatorname{ord}(1 / 2)(a \gamma)$ and hence $\sigma \in \mathrm{o}$. If $\sigma$ is a unit,

$$
E\left(u, \alpha \sigma^{-1} r\right) \varphi(z)=\beta v+z .
$$

Similarly, the coefficient of $v$ can be made zero and we obtain an isometry fixing both $w$ and $z$. If, however, $\sigma$ is not a unit, then $\gamma q(r)$ is a unit (since $B(r, z)$ is a unit). In $\Psi(r) \varphi(z)$ the new coefficient of $r$ becomes $\sigma q(r)^{-1}$. Now proceed as before. Note that if ord (2a) is even, $\Psi(r)$ can be expressed in terms of the elements of $\mathscr{E}$ and $O(H)$. This completes the reduction.

2.5. Let $L=\mathfrak{o} w \perp(\mathfrak{o} x+\mathfrak{o} y)=\langle a\rangle \perp\langle A(b, 2 \zeta)\rangle$ with ord $b$ odd. Then $O(M)$ is generated by $O(H)$ and $\mathscr{E}$, together with one symmetry $\Psi(r)$ where ord $(q(r))$ is odd.

Proof. Let $\varphi \in O(M)$. Since $B(w+x, y)=1$ and $q(y) \in \mathrm{D}$, by Remark 2.2, $\varphi$ can be assumed to have the property $\varphi(w+x)=$ $w+x$. But

$$
L=\mathfrak{o}(w+x) \perp(\mathfrak{o}(a y-w)+\mathfrak{o}(a x-b w)) .
$$

The result now follows from 2.4 since $B(a y-w, a x-b w)$ is a unit, $2 q(a y-w)=a(1+2 a \zeta)$ and $2 q(a x-b w)=a b(a+b)$.

2.6. Let $L=(\mathfrak{o} w+\mathfrak{o} z) \perp(\mathfrak{o} x+\mathfrak{o} y)=\langle A(a, c)\rangle \perp\langle A(b, 2 \zeta)\rangle$ with ord $(a b)$ odd. Then $O(M)$ is generated by $O(H)$ and $\mathscr{E}$, together with one symmetry $\Psi(r)$ where ord $(q(r))$ is odd.

Proof. Let $\varphi \in O(M)$. By Remark 2.2, we may assume $\varphi(x)=$ $x$. If it can also be arranged that $\varphi(y)=y$, invoking 2.4 will complete the proof. Changing $z$ if necessary, we may assume that either $c \in 20$ or ord $(a c)$ is odd.

If $c \in 20$, using 2.2 again, we also have $\varphi(w)=w$. When $c \in 20$, let $g=\operatorname{ord}\left(2 b^{-1}\right)$ and $h=[(g+1) / 2]$. Now put $s=\pi^{h}(x-b y)$ so that $q(s) \in \mathfrak{0}$. If, however, $c \notin 20$ so that $\operatorname{ord}(a c) \equiv \operatorname{ord}(a b) \bmod 2$, let $2 h=$ ord $\left(c b^{-1}\right) \geqq 0$. Since ord $(a c)$ is odd, there exists $r \in \mathfrak{o} w+\mathfrak{o} z$ such that

$$
s=r+\pi^{h}(x-b y)
$$


is in $M_{*}$. Moreover, $B(s, w)$ is a unit, and by Remark 2.2 we can change $\varphi$ so that again $\varphi(w)=w$.

Examining the proof of Proposition 1.4(1), we find that either $\varphi$ or $\Delta \varphi$ can be expressed in the form $E\left(u, p_{1}\right) E\left(v, p_{2}\right) \Phi(\varepsilon) \theta$ where $\theta \in O(L)$ and $B\left(p_{i}, x\right)=B\left(p_{i}, w\right)=0$ for $i=1,2$ (since the conditions $\varphi(x)=x$ and $\varphi(w)=w$ ensure that the component of $\varphi(v)$ in $H$ is primitive). To prove 2.6 it now suffices to show that any $\varphi \in O(L)$ with $\varphi(x)=x$ and $\varphi(w)=w$ can be expressed in terms of the given generators.

We still have $h$ and $s$ available as constructed. In both cases,

$$
E(u, s)(y)=\alpha u+y
$$

where $\alpha=B(s, y)=\pi^{h}(1-2 b \zeta)$. Note that $E(u, s)$ leaves $x$ fixed. Let

$$
\varphi(\alpha u+y)=\alpha u+\beta(w-a z)+\gamma(x-b y)+y
$$

where $\beta, \gamma \in \mathfrak{D}$. Since $q(y)=q(\varphi(\alpha u+y))$, it follows that $a \beta^{2}+b \gamma^{2} \in$ $20+a c 0$, and hence ord $\beta \geqq h$ and ord $\gamma \geqq h$ (in fact, ord $\beta \geqq h+1$ if $c \notin 20)$. Thus

$$
\varphi(\alpha u+y)=\alpha u+\pi^{h} t+y
$$

where $t \in L$ and $B(t, x)=0$. Suppose that $q(t) \in \mathfrak{0}$. Then

$$
E(u,-s) E\left(v, \alpha^{-1} \pi^{h} t\right) \varphi E(u, s)(y)=y
$$

and changing $\varphi$ by elements in $O(H)$ and $\mathscr{E}$ we have obtained an isometry acting identically on $x$ and $y$. This, by 2.4, would complete the proof. If $c \notin 20$ we need one symmetry in 2.4; this is also true if $c \in 20$ and ord $(2 a)$ is odd. When $c \in 20$ and ord $(2 a)$ is even, the symmetry will appear below.

It remains to show $q(t) \in \mathfrak{D}$. Since

$$
\pi^{2 h} q(t)=-\pi^{h} B(t, y)=\gamma(1-2 b \zeta),
$$

it suffices to show ord $\gamma \geqq 2 h$. Again, from $q(y)=q(\varphi(\alpha u+y))$,

$$
a \beta^{2}+b \gamma^{2}+2 \gamma \equiv\left\{\begin{array}{lll}
0 \bmod b \pi^{4 h} & \text { if } \quad c \notin 20 \\
0 \bmod 2 \pi^{2 h} & \text { if } \quad c \in 20 .
\end{array}\right.
$$

Except when $c \in 20$, ord $\left(2 b^{-1}\right)=2 h-1=$ ord $\gamma$ and $2+b \gamma \equiv 0 \bmod 2 \pi$, we can conclude that ord $\gamma \geqq 2 h$. In the exceptional case, replace $\varphi$ by $\Psi(x-b y) \varphi$ and the new coefficient of $x$ (the new $\gamma$ ) is divisible by $\pi^{2 h}$. This completes the proof.

THEOREM 2.7. Let $M$ be a unimodular o-lattice split by a hyperbolic plane $H$. Then the orthogonal group $O(M)$ is generated by 
$O(H)$ and $\mathscr{E}$, together with at most one symmetry $\Psi(r)$. The symmetry $\Psi(r)$ is required if and only if $M$ contains an element $r$ with

$$
\text { ord }\left(q(r)^{-1}\right) \geqq 1
$$

and odd.

Proof. This merely summarizes the results 2.3-2.6.

CoROllary 2.8. Any element $\varphi \in O(M)$ can be expressed in the form

$$
\varphi=\Delta^{c} \Phi(\varepsilon) \Psi(r)^{d} \theta
$$

where $c, d \in\{0,1\}, \varepsilon$ is a unit and $\theta \in \mathscr{E}$. In particular, $d=0$ if $M$ does not contain an element $r$ with ord $\left(q(r)^{-1}\right) \geqq 1$ and odd.

Proof. This follows immediately from Theorem 2.7 and Proposition 1.1 .

Let $\Theta$ denote the spinor norm on the special orthogonal group $S O(V)$ and $S k(M)$ the spinorial kernel in $O(M)$,

$$
S k(M)=\{\varphi \in S O(M) \mid \Theta(\varphi)=1\} .
$$

THEOREM 2.9. Let $M=H \perp K$ and assume $q(K)$ contains a unit of $\mathrm{D}$. Then

$$
\operatorname{Sk}(M)=\mathscr{E}
$$

Proof. It is well-known that the isometry $E(p, s)$ has spinor norm 1. Hence $\mathscr{E} \subseteq S k(M)$ always. Conversely, let

$$
\varphi=\Delta^{c} \Phi(\varepsilon) \Psi(r)^{d} \theta
$$

have spinor norm 1. Then $\operatorname{det} \varphi=1$ gives $c=d$. Now $\Theta(\varphi)=$ $(-q(r))^{\circ} \varepsilon$, since $\Delta=\Psi(u-v)$ and $\Phi(\varepsilon)=\Delta \Psi(u-\varepsilon v)$. If $r$ exists, ord $(q(r))$ is odd, and hence $c=0$ and $\varepsilon=\eta^{2}$ for some unit $\eta$. Corollary 1.3 now gives $S k(M) \subseteq \mathscr{E}$.

3. E्E-invariant sublattices. We now study the $\mathscr{E}$-invariant sublattices of $M$, that is, sublattices of $M$ that are invariant under the action of $\mathscr{E}$. For $O(M)$-invariant sublattices, see [11].

Clearly, $M_{*}=\{s \in M \mid q(s) \in \mathfrak{D}\}$ is invariant under the action of both $O(M)$ and $\mathscr{E}$. Let $M^{*}$ be the dual lattice of $M_{*}$, that is,

$$
M^{*}=\left\{s \in V \mid B\left(s, M_{*}\right) \subseteq \mathrm{p}\right\} .
$$


Then $2 M^{*} \leqq M$ and $2 M^{*}$ is invariant under the action of $O(M)$. Let $P$ be a sublattice of $M$. Define

$$
a(P)=\sum_{r \in P} B\left(r, M_{*}\right) .
$$

Then $B\left(P, M_{*}\right) \subseteq \mathfrak{a}(P)$ and $\mathfrak{a}(P)$ is an ideal in $\mathfrak{b}$, since each $B\left(r, M_{*}\right)$ is.

THEOREM 3.1. Let $M=H \perp K$ where $q(K)$ contains a unit. If card $\mathfrak{o} / \mathfrak{p}=2$, assume also $r(M) \geqq 7$. Then a sublattice $P$ of $M$ is $\mathscr{E}$-invariant if and only if

$$
M_{*} \subseteq \mathfrak{a}(P)^{-1} P \subseteq M^{*} .
$$

Proof. Write $a=\mathfrak{a}(P)$. Since $B\left(\mathfrak{a}^{-1} P, M_{*}\right) \subseteq \mathfrak{0}$, it follows that $\mathfrak{a}^{-1} P \subseteq M^{*}$. Now let $x \in K_{*}$ and $y \in P$. Since $B\left(P, M_{*}\right) \subseteq \mathfrak{a}$,

$$
E(u, x)(y) \equiv y \bmod a M_{*},
$$

and $P$ is $\mathscr{E}$-invariant if $a M_{*} \subseteq P$. It remains to show that if $r \in P$ and $B\left(r, M_{*}\right)=\mathfrak{b}$, then $\mathfrak{b} M_{*} \subseteq P$ if $P$ is $\mathscr{E}$-invariant.

Write $r=\beta u+\gamma v+s$ where $s \in K$. Then $\mathfrak{b}=\beta \mathfrak{D}+\gamma_{\mathfrak{D}}+B(s$, $K_{*}$ ). We may assume $\mathfrak{b}=\beta \mathfrak{b}$ (otherwise replace $r$ by $E(u, t)(r)$ where $B(s, t)$ generates $\mathfrak{b}$, or interchange $u$ and $v$ ). Take $y \in K_{*}$ such that $q(y)$ is a unit and, when card $o / p=2$, also $B(s, y)=0$. For a suitable unit $\varepsilon,(E(v, \varepsilon y)-I)(r)$ gives rise to an element $v+z$ in $\mathfrak{b}^{-1} P$ with $z \in K$ and $q(z)$ a unit. Then, for any unit $\eta$,

$$
(E(u, \eta z)-I)(v+z)=-\eta z+\eta(2-\eta) q(z) u
$$

is in $\mathfrak{b}^{-1} P$. If card $\mathfrak{o} / \mathfrak{p} \geqq 4$, it follows that $u \in \mathfrak{b}^{-1} P$ and it is now easy to show that $M_{*} \subseteq \mathfrak{b}^{-1} P$. If card $\mathfrak{o} / \mathfrak{p}=2$, put $\eta=1$ so that $v+q(z) u$ is in $\mathfrak{b}^{-1} P$. Take $p \in K$ primitive and isotropic. Then $E(u$, $p)(v+q(z) u)$ is in $\mathfrak{b}^{-1} P$. Hence $p \in \mathfrak{b}^{-1} P$ and consequently $M_{*} \subseteq \mathfrak{b}^{-1} P$. This completes the proof.

CoRollary 3.2. Let $r \in M$ and $B\left(r, M_{*}\right)=a$. Under the hypotheses of the theorem, $a M_{*}+\mathfrak{o} r$ is the smallest $\mathscr{E}$-invariant sublattice in $M$ containing $r$.

\section{Proof. Clear.}

Introduce an indexing set $\Xi$ so that the lattices $M_{\xi}, \xi \in \Xi$, are all the distinct lattices on $V$ satisfying

$$
M_{*} \subseteq M_{\xi} \subseteq M^{*} \text {. }
$$

If $\mathfrak{o} / \mathfrak{p}$ is finite, then $\Xi$ is also finite. Let $a$ be an ideal such that $\mathfrak{a} M_{\xi} \subseteq$ 
M. Then $a M_{\xi}$ is an $\mathscr{E}$-invariant lattice.

4. The subgroups $\mathscr{E}\left(\mathfrak{a} M_{\xi}\right)$ and $\mathscr{F}\left(\mathfrak{a} M_{\xi}\right)$. For $\xi \in \Xi$, let $\mathfrak{a}$ be an ideal such that $\mathfrak{a} M_{\xi} \subseteq M_{*}$. Define $\mathscr{E}\left(\mathfrak{a} M_{\xi}\right)$ to be the subgroup of $O(M)$ generated by isometries of the form $\psi E(u, z) \psi^{-1}$ and $\psi E(v, z) \psi^{-1}$ where $\psi \in \mathscr{E}$ and $z \in K \cap a M_{\xi}$. The subgroups $\mathscr{E}\left(\mathfrak{a} M_{\xi}\right)$ are obviously normalized by $\mathscr{E}$. Also, let

$$
\mathscr{F}\left(\mathfrak{a} M_{\hat{\xi}}\right)=\left\{\varphi \in O(M) \mid[\varphi, \mathscr{E}] \subseteq \mathscr{E}\left(\mathfrak{a} M_{\xi}\right)\right\} .
$$

Then any subgroup $\mathscr{N}$ of $O(M)$ satisfying

$$
\mathscr{E}\left(\mathfrak{a} M_{\hat{\xi}}\right) \leqq \mathscr{N} \leqq \mathscr{F}\left(\mathfrak{a} M_{\xi}\right)
$$

for some $a M_{\xi} \subseteq M_{*}$ is normalized by $\mathscr{E}$ since

$$
[\mathscr{N}, \mathscr{E}] \subseteq\left[\mathscr{F}\left(a M_{\xi}\right), \mathscr{E}\right] \subseteq \mathscr{E}\left(\mathfrak{a} M_{\xi}\right) \subseteq \mathscr{N} \text {. }
$$

For $a M_{\xi} \subseteq M_{*}$ define the congruence subgroup $O\left(\mathfrak{a} M_{\xi}\right)$ by $O\left(a M_{\xi}\right)=$ $\left\{\varphi \in O(M) \mid \varphi(x) \equiv x \bmod a M_{\xi}\right.$ for all $\left.x \in M_{*}\right\} \times\{ \pm I\}$. These subgroups are normalized by $\mathscr{E}$ since $M_{*}$ and $\mathfrak{a} M_{\xi}$ are $\mathscr{E}$-invariant. If $\mathfrak{a} M_{\xi}=$ $M_{*}$, then $O\left(M_{*}\right)=O(M)$. Now let $a \subseteq \mathfrak{p}$. If $\varepsilon \equiv 1 \bmod a$, then $\Phi(\varepsilon) \in$ $O\left(\mathfrak{a} M_{\xi}\right)$. Also, for $z \in K \cap \mathfrak{a} M_{\xi}$, both $E(u, z)$ and $E(v, z)$ are in $O\left(\mathfrak{a} M_{\xi}\right)$ provided $\mathfrak{a} q\left(M_{\xi}\right) \subseteq \mathfrak{D}$. Hence $\mathscr{E}\left(\mathfrak{a} M_{\xi}\right) \subseteq O\left(\mathfrak{a} M_{\xi}\right)$, provided $\mathfrak{a} q\left(M_{\xi}\right) \subseteq \mathfrak{D}$.

Lemma 4.1. Let $\mathfrak{a} \leqq \mathfrak{p}$ and $\mathfrak{a} q\left(M_{\xi}\right) \subseteq \mathfrak{0}$. Then $\varphi \in O\left(\mathfrak{a} M_{\xi}\right)$ can be expressed in the form

$$
\varphi= \pm E(u, x) E(v, y) \Phi(\varepsilon) \theta
$$

where $x$ and $y$ are in $K \cap a M_{\xi}, \varepsilon \equiv 1 \bmod a$ and $\theta \in O(K) \cap O\left(a M_{\xi}\right)$.

Proof. Let $\varphi(v)=\alpha u+\beta v+s$ where $s \in K \cap a M_{\xi}$ and

$$
\beta \equiv \pm 1 \bmod a \text {. }
$$

If $\beta \equiv-1 \bmod a$, replace $\varphi$ by $-\varphi$. Now put $\phi_{1}=\Phi(\beta) E\left(u, \beta^{-1} s\right) \varphi \epsilon$ $O\left(\mathfrak{a} M_{\xi}\right)$ so that $\varphi_{1}(v)=v$. Let $\varphi_{1}(u)=u-q(t) v+t$ where $t \in K \cap \mathfrak{a} M_{\xi}$. Put $\theta=E(v, t) \varphi_{1} \in O\left(a M_{\xi}\right)$. Then $\theta \in O(K)$ and $\varphi$ can be rewritten in the desired form.

THEOREM 4.2. Let $M=H \perp K$ where $q(K)$ contains a unit. If card $\mathfrak{o} / \mathfrak{p}=2$, assume that $r(M) \geqq 9$. Then, if $\mathfrak{a} q\left(M_{\xi}\right) \leqq \mathfrak{v}$,

$$
\mathscr{E}\left(\mathfrak{a} M_{\xi}\right)=\left[O\left(\mathfrak{a} M_{\xi}\right), \mathscr{E}\right]
$$

and hence

$$
\mathscr{E}\left(a M_{\xi}\right) \subseteq O\left(a M_{\xi}\right) \subseteq \mathscr{F}\left(a M_{\xi}\right)
$$


Proof. We first show that $\left[O\left(\mathfrak{a} M_{\xi}\right), \mathscr{E}\right] \leqq \mathscr{E}\left(\mathfrak{a} M_{\xi}\right)$. If $a M_{\xi}=M_{*}$, then $\mathscr{E}\left(M_{*}\right)=\mathscr{E}$ and $[O(M), \mathscr{E}] \subseteq \mathscr{E}$ by Corollary 2.8. Now assume $\mathfrak{a} \subseteq \mathfrak{p}$. Consider first $[\varphi, E(u, t)]$ where $\varphi \in O\left(a M_{\xi}\right)$ and $t \in M_{*}$. By Lemma 4.1,

$$
\varphi= \pm E(u, x) E(v, y) \Phi(\varepsilon) \theta
$$

with $E(u, x)$ and $E(v, y)$ in $\mathscr{E}\left(\mathfrak{a} M_{\xi}\right)$. From Proposition 1.1,

$$
[\varphi, E(u, t)] \equiv E(u, \varepsilon \theta(t)-t) \bmod \mathscr{E}\left(a M_{\xi}\right) \text {. }
$$

But $[\varphi, E(u, t)]$ is in $O\left(a M_{\xi}\right)$ and hence

$$
E(u, \varepsilon \theta(t)-t)(v) \equiv v \bmod a M_{\xi}
$$

so that $\varepsilon \theta(t)-t \in \mathfrak{a} M_{\xi}$. Hence $[\varphi, E(u, t)] \in \mathscr{E}\left(\mathfrak{a} M_{\xi}\right)$. From the properties of commutators, it follows that

$$
\left[O\left(a M_{\xi}\right), \mathscr{E}\right] \subseteq \mathscr{E}\left(a M_{\xi}\right) .
$$

For the converse inclusion we must show $E(u, z)$ and $E(v, z)$ are in $\left[O\left(a M_{\xi}\right), \mathscr{E}\right]$ for all $z \in K \cap \mathfrak{a} M_{\xi}$. If card $\mathfrak{o} / \mathfrak{p} \geqq 4$, there exists a unit $\zeta$ such that $\eta^{-1}=\zeta^{2}-1$ is also a unit. Then

$$
E(u, z)=\left[\Phi\left(\zeta^{2}\right), E(u, \eta z)\right] \in\left[\mathscr{E}, O\left(a M_{\xi}\right)\right] .
$$

Finally, let $\operatorname{card} \mathrm{o} / \mathfrak{p}=2$. Since now $r(M) \geqq 9, M=H \perp H^{\prime} \perp K^{\prime}$ where $z \in K^{\prime}$ and $H^{\prime}=\mathrm{o} u^{\prime}+\mathfrak{o} v^{\prime}$ is a second hyperbolic plane. Then

$$
\text { [ } \begin{aligned}
\left.E\left(u^{\prime}, z\right), E\left(v^{\prime}, u\right)\right] & =E\left(E\left(u^{\prime}, z\right)\left(v^{\prime}\right), u\right) E\left(v^{\prime},-u\right) \\
& =E\left(u,-E\left(u^{\prime}, z\right)\left(v^{\prime}\right)\right) E\left(u, v^{\prime}\right) \\
& =E\left(u, z+q(z) u^{\prime}\right)
\end{aligned}
$$

is in $\left[O\left(\mathfrak{a} M_{\xi}\right], \mathscr{E}\right]$. Since $a q\left(M_{\xi}\right) \subseteq \mathfrak{D}$, we have $q(z) u^{\prime} \in \mathfrak{a} M_{\xi}$. A similar argument shows that $E\left(u, q(z) u^{\prime}\right)$ is also in $\left[O\left(a M_{\xi}\right), \mathscr{E}\right]$. The result now follows immediately.

THEOREM 4.3. Let $M=H \perp K$ where $q(K)$ contains a unit. If card $\mathfrak{o} / \mathfrak{p}=2$, assume that $r(M) \geqq 9$. Then

$$
\mathscr{E}=\Omega(M) \text {. }
$$

Proof. Take $a M_{\xi}=M_{*}$ in Theorem 4.2. Then

$$
\mathscr{E}=\mathscr{E}\left(M_{*}\right)=[O(M), \mathscr{E}] \subseteq \Omega(M) \subseteq \mathscr{E},
$$

the final inclusion following from Theorem 2.9.

REMARK 4.4. With greater effort, a stronger result may be 
obtained when card $\mathfrak{o} / \mathfrak{p}=2$ (see, for example, [9; Theorem 2.6]). Also, when $a q\left(M_{\xi}\right) \nsubseteq \mathfrak{D}$, anormalous behaviour may occur (see [10; Table II]).

5. Subgroups normalized by $\Omega(M)$. Let $\mathscr{N}$ denote a subgroup of $O(M)$ normalized by $\mathscr{E}$. We now prove, under suitable hypotheses, that there exists a sublattice $a M_{\xi}$ invariant under $\mathscr{E}$ such that

$$
\mathscr{E}\left(a M_{\xi}\right) \subseteq \mathscr{N} \leqq \mathscr{F}\left(a M_{\xi}\right)
$$

The method is as follows. Assume $\varphi \in \mathscr{N}$ and choose $\psi \in \mathscr{E}$ as in Proposition 1.4 such that

$$
\psi \varphi \psi^{-1}=E(u, x) E(v, y) \Phi(\varepsilon) \theta
$$

is also in $\mathscr{r}$. We shall show that $E(u, x), E(v, y)$ and $\Phi(\varepsilon) \theta$ are all in $\mathscr{N}$ and use these isometries (varying $\varphi$ in $\mathscr{N}$ ) to obtain a maximal subgroup of the form $\mathscr{E}\left(\mathfrak{a} M_{\xi}\right)$ in $\mathscr{N}$. It then remains to prove $\mathscr{N} \cong$ $\mathscr{F}\left(a M_{\xi}\right)$. We prepare for this theorem with a number of lemmas.

Lemma 5.1. Let $M=H \perp K$ where $q(K)$ contains a unit. Then if card $\mathfrak{o} / \mathfrak{p} \geqq 8$ and

$$
\varphi=E(u, x) E(v, y) \Phi(\varepsilon) \theta
$$

is in a subgroup $\mathscr{N}$ normalized by $\mathscr{E}$, there exist units $\zeta$ and $\eta$ (independent of $\varphi$ ) such that $E(u, \zeta x)$ and $E(v, \eta y)$ are also in $\mathscr{N}$.

Proof. Modify Lemma 3.8 in [9].

Lemma 5.2. Assume $r(M) \geqq 7$ and $E(u, x)$ is in $\mathscr{N}$. Then $E(u, \alpha x)$ is in $\mathscr{N}$ for all $\alpha \in \mathfrak{D}$.

Proof. $x \in K$ can be embedded in a binary (or unary) sublattice $B$ of $K$ with $K=B \perp C$. Then $r(C) \geqq 3$. From [14; 93: 20], $\Theta(S O(C)$ ) contains all units. Let $\varepsilon$ be any unit and take $\theta \in S O(C)$ such that $\Theta(\theta)=\varepsilon$. Then $\Phi(\varepsilon) \theta \in S k(M)=\mathscr{E}$. Conjugating $E(u, x)$ in $\mathscr{N}$ with $\Phi(\varepsilon) \theta$ gives $E(u, \varepsilon \theta(x))=E(u, \varepsilon x)$ is in $\mathscr{N}$ for all units $\varepsilon$. If $\alpha \in \mathfrak{p}$ is not a unit, then $\alpha=1+\varepsilon$ with $\varepsilon$ unit and now $E(u, \alpha x)$ is also in $\mathscr{N}$. This proves the lemma.

The previous two lemmas show that for $r(M) \geqq 7$ and card $\mathrm{o} / \mathfrak{p} \geqq$ 8 that if $E(u, x) E(v, y) \Phi(\varepsilon) \theta$ lies in a subgroup $\mathscr{N}$ normalized by $\mathscr{E}$, then so do $E(u, x), E(v, y)$ and $\Phi(\varepsilon) \theta$. We show now that this is still true for card $\mathfrak{o} / \mathfrak{p}=2$ or 4 provided the rank of $M$ is at least 9 .

Lemma 5.3. Let $M=H \perp K$ with $r(M) \geqq 9$ and $\varphi=E(u, x) E(v$, 
$y) \Phi(\varepsilon) \theta \in \mathscr{N}$ where $x, y \in K_{*}$ and $\theta \in O(K)$. Then $E(u, x), E(v, y)$ and $\Phi(\varepsilon) \theta$ are all in $\mathscr{N}$.

Proof. Since $r(M) \geqq 9$, we have $M=H \perp H^{\prime} \perp K^{\prime}$ where $H^{\prime}=$ $\mathfrak{v} u^{\prime}+\mathfrak{o} v^{\prime}$ is a hyperbolic plane and $y \in K^{\prime}$. Then

$$
E(u,-x)\left[\rho, E\left(u, u^{\prime}\right)\right] E(u, x)=E(v, y) E\left(u, \varepsilon \theta\left(u^{\prime}\right)\right) E(v,-y) E\left(u,-u^{\prime}\right)
$$

is in $\mathscr{N}$. Hence

$$
\begin{aligned}
& E\left(u, \varepsilon \theta\left(u^{\prime}\right)\right) E\left(E(v,-y)(u),-u^{\prime}\right) \\
& \quad=E\left(u, \varepsilon \theta\left(u^{\prime}\right)\right) E\left(u+y-q(y) v,-u^{\prime}\right) \\
& \quad=E\left(u, \varepsilon \theta\left(u^{\prime}\right)-u^{\prime}\right) E\left(u^{\prime}, y-q(y) v\right)
\end{aligned}
$$

is also in $\mathscr{N}$. Let $t=\varepsilon \theta\left(u^{\prime}\right)-u^{\prime}$. Take $s \in K^{\prime}$ with $q(s)$ a unit and $B(s, y)=0$. Then $\left[E(u, s), E(u, t) E\left(u^{\prime}, y-q(y) v\right]\right.$, and hence also $E\left(u^{\prime}, q(y)(s+q(s) u)\right)$, are in $\mathscr{N}$. But $\mathrm{o} v+\mathfrak{o}(s+q(s) u)$ is a hyperbolic plane, so that both $E\left(u^{\prime}, q(y) v\right)$ and $E(u, t) E\left(u^{\prime}, y\right)$ are in $\mathscr{N}$. This already completes the proof in the special case where $\varepsilon=1$ and $\theta$ is the identity map, since then $t=0$. Returning to the general case, since $r\left(K^{\prime}\right) \geqq 5$, there exists $\psi \in O\left(K^{\prime}\right)$ such that $\psi(y)=y$ and $\Delta \psi \in$ $\mathscr{E}$. Conjugating $E(u, t) E\left(u^{\prime}, y\right)$ with $\Delta \psi$, shows that $E(v, \psi(t)) E\left(u^{\prime}, y\right)$ is in $\mathscr{N}$. Hence $E(u, t) E(v,-\psi(t)) \in \mathscr{N}$ and, by the special case noted above, it follows that $E(u, t)$ is in $\mathscr{N}$. Finally, $E\left(u^{\prime}, y\right) \in \mathscr{N}$ and the result now follows.

Lemma 5.4. Let $M=H \perp K$ with $r(M) \geqq 7$. If $\operatorname{card} \mathfrak{o} / \mathfrak{p} \leqq 4$, assume also $r(M) \geqq 9$. Let $E(u, x) \in \mathscr{N}$ where $x \in K_{*}$ and $B\left(x, M_{*}\right)=$ a. Then

$$
\mathscr{E}\left(a M_{*}\right) \leqq \mathscr{N}
$$

Proof. Take $z \in K_{*}$ such that $B(x, z)=\alpha$ where $\mathrm{o} \alpha=\mathfrak{a}$. We may assume $q(z)$ is a unit, for if not, take $z_{1} \in K_{*}$ with $B\left(z, z_{1}\right)=0$ and $q\left(z_{1}\right)$ a unit; if $B\left(x, z_{1}\right) \in \alpha \mathfrak{u}$, replace $z$ by $z_{1}$, otherwise, replace $z$ by $z+z_{1}$. Moreover, there exists $y \in K_{*}$ with $B(x, y)=0$ and $q(y)$ a unit. Let $\varepsilon=q(z) q(y)$. Conjugating $E(u, x) \in \mathscr{N}$ with $\Phi(\varepsilon) \Psi(z) \Psi(y)$ from $S k(M)=\mathscr{E}$ gives $E\left(\varepsilon u, x-\alpha q(z)^{-1} z\right) \in \mathscr{N}$. From Lemma 5.2 it follows that $E(u, \alpha z)$ is in $\mathscr{N}$. If $w \in K_{*}$ and card $\mathfrak{v} / \mathfrak{p} \geqq 8$, there is a unit $\eta$ such that $q(z+\eta w) \in \mathfrak{u}$ and $B(x, z+\eta w) \in \alpha \mathfrak{u}$. A similar argument shows $E(u, \alpha(z+\eta w))$, and hence also $E(u, \alpha w)$, are in $\mathscr{N}$. Conjugating with $\Delta \Phi(-q(z)) \Psi(z) \in \mathscr{E}$ gives now $\mathscr{E}\left(\mathfrak{a} M_{*}\right) \subseteq \mathscr{N}$.

Now assume card $\mathfrak{o} / \mathfrak{p} \leqq 4$ so that $r(M) \geqq 9$. Then $M=H \perp H^{\prime} \perp K^{\prime}$ with $x \in K^{\prime}$. Conjugating $E(u, x)$ with $E\left(u^{\prime}, z\right)$ leads to $E\left(u, \alpha u^{\prime}\right) \in$ $\mathscr{N}$. Similarly, $E\left(u, \alpha v^{\prime}\right) \in \mathscr{N}$. Take $t \in K_{*}^{\prime}$. Finally, conjugating 
$E\left(u, \alpha u^{\prime}\right)$ with $E\left(v^{\prime}, t\right) \in \mathscr{E}$ shows that $E(u, \alpha t)$ is in $\mathscr{N}$ and hence again, $\mathscr{E}\left(a M_{*}\right) \subseteq \mathscr{N}$.

THEOREM 5.5. Let $M$ be a unimodular lattice with $r(M) \geqq 7$, and $r(M) \geqq 9$ if card $\mathfrak{o} / \mathfrak{p}=2,4$. Then a subgroup $\mathscr{N}$ of the orthogonal group $O(M)$ is normalized by the commutator subgroup $\Omega(M)$ if and only if it satisfies

$$
\mathscr{E}\left(\mathfrak{a} M_{\xi}\right) \subseteq \mathscr{N} \subseteq \mathscr{F}\left(\mathfrak{a} M_{\xi}\right)
$$

for some ideal a in $\mathrm{o}$ and an invariant sublattice $M_{\xi}$ with $\mathfrak{a} M_{\xi} \subseteq M_{*}$.

Proof. We have already observed that subgroups satisfying these ladder relations are normalized by $\mathscr{E}=\Omega(M)$. Now assume $\mathscr{N}$ is a subgroup normalized by $\Omega(M)$ and choose $a M_{\xi} \subseteq M_{*}$ maximal such that $\mathscr{E}\left(\mathfrak{a} M_{\xi}\right) \subseteq \mathscr{N}$. Clearly, at least $\{I\}=\mathscr{E}\left(\{O\} M_{*}\right) \subseteq \mathscr{N} ;$ moreover, if both $\mathscr{E}\left(a_{1} M_{\xi_{1}}\right)$ and $\mathscr{E}\left(a_{2} M_{\xi_{2}}\right)$ are contained in $\mathscr{N}$, these two subgroups generate $\mathscr{E}\left(a_{3} M_{\xi_{3}}\right) \leqq \mathscr{N}$ where $\mathfrak{a}_{3}=a_{1}+\mathfrak{a}_{2}$ (see $\S 4$ ).

Now let $\varphi \in \mathscr{N}$; we must prove $\varphi \in \mathscr{F}\left(\mathfrak{a} M_{\xi}\right)$. By Proposition 1.4 there exists $\psi \in \Omega(M)$ such that

$$
\psi \varphi \psi^{-1}=E(u, x) E(v, y) \Phi(\varepsilon) \theta
$$

where $\theta \in O(K)$. By Lemmas 5.1-5.3 we know that $E(u, x)$ and $E(v$, $y)$ are in $\mathscr{N}$ and hence by Lemma 5.4 and $\S 4$ they are even in $\mathscr{E}\left(a M_{\xi}\right)$. It therefore suffices to prove that $\Phi(\varepsilon) \theta$ is in $\mathscr{F}\left(\mathfrak{a} M_{\xi}\right)$. For $s \in K_{*}$,

$$
[\Phi(\varepsilon) \theta, E(u, s)]=E(u, \varepsilon \theta(s)-s)
$$

is in $\mathscr{N}$. Again, from Lemma 5.4 and $\S 4$, it follows that $[\Phi(\varepsilon) \theta$, $E(u, s)]$ is in $\mathscr{E}\left(\mathfrak{a} M_{\xi}\right)$. Hence

$$
[\Phi(\varepsilon) \theta, \mathscr{E}] \subseteq \mathscr{E}\left(a M_{\xi}\right)
$$

and, therefore, $\Phi(\varepsilon) \theta \in \mathscr{F}\left(\mathfrak{a} M_{\xi}\right)$. This proves the theorem.

\section{REFERENCES}

1. A. Bak, On modules with quadratic forms, Lecture Notes in Mathematics 108, Springer, Berlin-Heidelberg-New York, 1969.

2. C. Chang, Local Orthogonal Groups over 2-adic Fields, Ph. D. Dissertation, University of Notre Dame, 1971.

3. L. E. Dickson, Linear Groups, Dover Edition, New York, 1958.

4. J. Dieudonné, La géométrie des Groupes Classiques, Springer, Berlin-GöttingenHeidelberg, 1963.

5. M. Eichler, Quadratische Formen und Orthogonale Gruppen, Springer, BerlinGöttingen-Heidelberg, 1952.

6. J. S. Hsia, Integral equivalence of vectors over local modular lattices, Pacific J. Math., 23 (1967), 527-542. 
7. D. G. James, On the orthogonal groups of lattices over local fields, J. Reine Angew. Math., 225 (1967), 116-119.

8. - On the structure of orthogonal groups over local rings, Amer. J. Math., 95 (1973), 255-265.

9. _ Orthogonal groups of dyadic unimodular quadratic forms, Math. Ann., 201 (1973), 65-74.

10. - The structure of orthogonal groups of 2-adic unimodular quadratic forms, J. Number Theory, 5 (1973), 444-455.

11. A. Kallmann, M. Kneser, U. Stuhler, Invariante Untermoduln quadratischer Moduln, J. Reine Angew. Math., 258 (1973), 51-54.

12. W. Klingenberg, Orthogonale Gruppen über lokalen Ringen, Amer. J. Math., 83 (1961), 281-320.

13. M. Kneser, Normal subgroups of integral orthogonal groups, Lecture Notes in Mathematics 108, Springer, Berlin-Heidelberg-New York, 1969.

14. O. T. O'Meara, Introduction to quadratic Forms, Springer, Berlin-HeidelbergNew York, 1963.

15. O.T.O'Meara, B. Pollak, Generation of local integral orthogonal groups II, Math. Z., 93 (1966), 171-188.

16. C. Riehm, Orthogonal groups over the integers of a local field, Amer. J. Math., 89 (1967), 549-577.

17. T. Tamagawa, On the structure of orthogonal groups, Amer. J. Math., 80 (1958), 191-197.

18. L. N. Vaserstein, Subgroups of finite index in Spin groups of rank $\geqq 2$, Mat. Sbornik, 75 (1968), 178-184.

19. C. T. C. Wall, On the orthogonal groups of unimodular quadratic forms II, J. Reine Angew. Math., 213 (1963) 122-136.

Received August 7, 1973. This research was partially supported by the National Science Foundation.

The Pennsylvania State University 



\section{PACIFIC JOURNAL OF MATHEMATICS}

EDITORS

RICHARD ARens (Managing Editor)

University of California

Los Angeles, California 90024

\section{R. A. Beaumont}

University of Washington

Seattle. Washington 98105

\section{J. DugundjI}

Department of Mathematics University of Southern California Los Angeles, California 90007

D. Gilbarg and J. Milgram

Stanford University

Stanford. California 94305

\section{ASSOCIATE EDITORS}
E. F. BECKENBACH
B. H. NEUMANN
F. WOLF
K. Yoshida

\section{SUPPORTING INSTITUTIONS}

\author{
UNIVERSITY OF BRITISH COLUMBIA \\ CALIFORNIA INSTITUTE OF TECHNOLOGY \\ UNIVERSITY OF CALIFORNIA \\ MONTANA STATE UNIVERSITY \\ UNIVERSITY OF NEVADA \\ NEW MEXICO STATE UNIVERSITY \\ OREGON STATE UNIVERSITY \\ UNIVERSITY OF OREGON \\ OSAKA UNIVERSITY
}

\author{
UNIVERSITY OF SOUTHERN CALIFORNIA \\ STANFORD UNIVERSITY \\ UNIVERSITY OF TOKYO \\ UNIVERSITY OF UTAH \\ WASHINGTON STATE UNIVERSITY \\ UNIVERSITY OF WASHINGTON

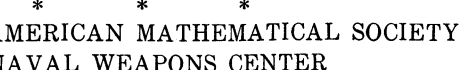

The Supporting Institutions listed above contribute to the cost of publication of this Journal, but they are not owners or publishers and have no responsibility for its content or policies.

Mathematical papers intended for publication in the Pacific Journal of Mathematics should be in typed form or offset-reproduced, (not dittoed), double spaced with large margins. Underline Greek letters in red, German in green, and script in blue. The first paragraph or two must be capable of being used separately as a synopsis of the entire paper. Items of the bibliography should not be cited there unless absolutely necessary, in which case they must be identified by author and Journal, rather than by item number. Manuscripts, in duplicate if possible, may be sent to any one of the four editors. Please classify according to the scheme of Math. Rev. Index to Vol. 39. All other communications to the editors should be addressed to the managing editor, or Elaine Barth, University of California, Los Angeles, California, 90024.

100 reprints are provided free for each article, only if page charges have been substantially paid Additional copies may be obtained at cost in multiples of 50 .

The Pacific of Journal Mathematics is issued monthly as of January 1966. Regular subscription rate: $\$ 72.00$ a year (6 Vols., 12 issues). Special rate: $\$ 36.00$ a year to individual members of supporting institutions.

Subscriptions, orders for back numbers, and changes of address should be sent to Pacific Journal of Mathematics, 103 Highland Boulevard, Berkeley, California, 94708.

\section{PUBLISHED BY PACIFIC JOURNAL OF MATHEMATICS, A NON-PROFIT CORPORATION}

Printed at Kokusai Bunken Insatsusha (International Academic Printing Co., Ltd.), 270, 3-chome Totsuka-cho. Shinjuku-ku, Tokyo 160. Japan.

Copyright (C) 1973 by Pacific Journal of Mathematics Manufactured and first issued in Japan 


\section{Pacific Journal of Mathematics}

\section{Vol. 52, No. $2 \quad$ February, 1974}

Harm Bart, Spectral properties of locally holomorphic vector-valued functions .....

J. Adrian (John) Bondy and Robert Louis Hemminger, Reconstructing infinite

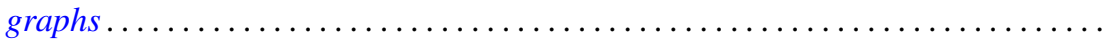

Bryan Edmund Cain and Richard J. Tondra, Biholomorphic approximation of planar

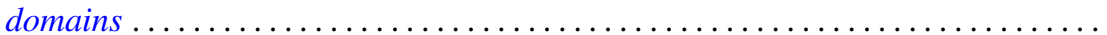

Richard Carey and Joel David Pincus, Eigenvalues of seminormal operators,

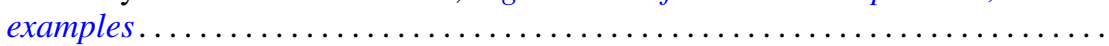

Tyrone Duncan, Absolute continuity for abstract Wiener spaces . . . . . . . . . . . . Joe Wayne Fisher and Louis Halle Rowen, An embedding of semiprime

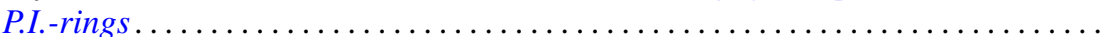

Andrew S. Geue, Precompact and collectively semi-precompact sets of semi-precompact continuous linear operators. . . . . . . . . . . . . . . .

Charles Lemuel Hagopian, Locally homeomorphic $\lambda$ connected plane continua ..... . Darald Joe Hartfiel, A study of convex sets of stochastic matrices induced by

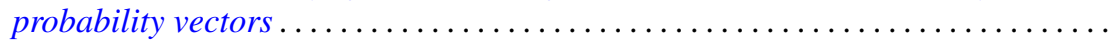

Yasunori Ishibashi, Some remarks on high order derivations $\ldots \ldots \ldots \ldots \ldots \ldots \ldots$ Donald Gordon James, Orthogonal groups of dyadic unimodular quadratic forms.

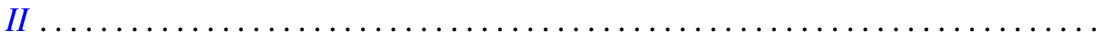

Geoffrey Thomas Jones, Projective pseudo-complemented semilattices . . . . . . . . . Darrell Conley Kent, Kelly Denis McKennon, G. Richardson and M. Schroder,

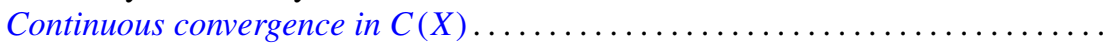

J. J. Koliha, Some convergence theorems in Banach algebras ...

Tsang Hai Kuo, Projections in the spaces of bounded linear oper

George Berry Leeman, Jr., A local estimate for typically real functions . .

475

Andrew Guy Markoe, A characterization of normal analytic spaces by the

homological codimension of the structure sheaf .........

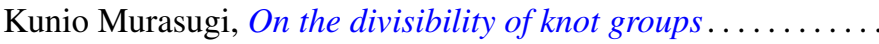

John Phillips, Perturbations of type I von Neumann algebras.

Billy E. Rhoades, Commutants of some quasi-Hausdorff matrices . .

David W. Roeder, Category theory applied to Pontryagin duality

Maxwell Alexander Rosenlicht, The nonminimality of the differential closure .

Peter Michael Rosenthal, On an inversion theorem for the general Mehler-Fock transform pair.

Alan Saleski, Stopping times for Bernoulli automorphisms

John Herman Scheuneman, Fundamental groups of compact complete locally affine complex surfaces. II. ........................

Vashishtha Narayan Singh, Reproducing kernels and operators with a cyclic vector. I. .

Peggy Strait, On the maximum and minimum of partial sums of random variables.

J. L. Brenner, Maximal ideals in the near ring of polynomials modulo 2 .

Ernst Gabor Straus, Remark on the preceding paper: "Ideals in near rings of polynomials over a field" ..........................

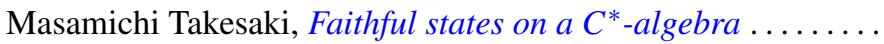

R. Michael Tanner, Some content maximizing properties of the regular simplex.

Andrew Bao-hwa Wang, An analogue of the Paley-Wiener theorem for certain

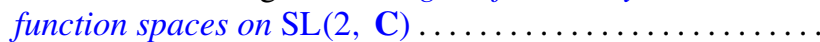

